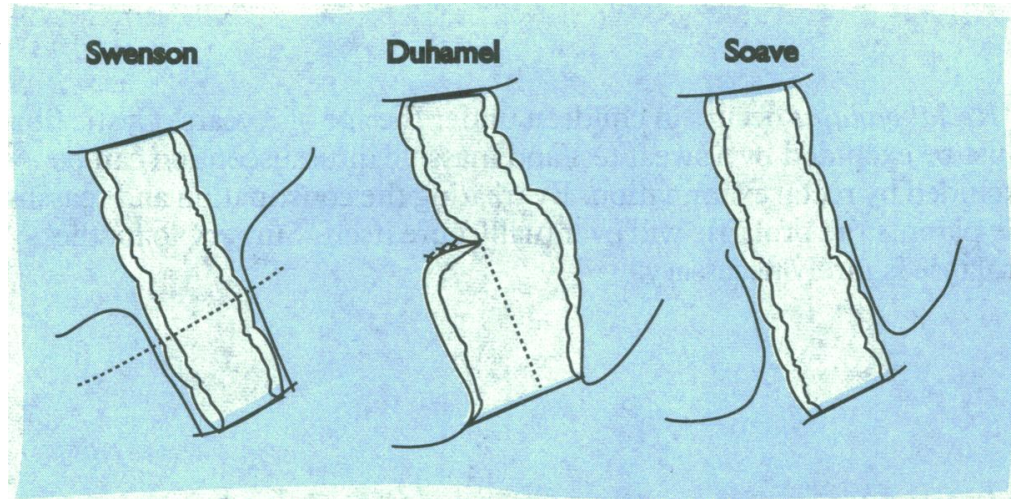

(a) Swenson operation - end to end anastomosis intussuscepting aganglionic and ganglionic bowel through anus and resection and anastomosis along dotted line. (b) Duhamel operation - anterior aganglionic and posterior ganglionic bowel. The dotted line indicates the staple closure and knife division between the two portions of bowel. (c) Soave operation-mucosa is removed, a muscular cuff of the aganglionic bowel is left and ganglionic bowel is pulled through to hang from the anus.

The photographs were prepared by the medical illustration department, North Manchester General Hospital.

\section{Complications}

The major complication and commonest cause of death in children with Hirschsprung's disease is enterocolitis which, although commoner in infancy, may occur in older children with untreated Hirschsprung's disease. It presents as a fulminating infection (often clostridial in origin) with toxaemia, abdominal distension, and diarrhoea. Despite treatment with antibiotics, intravenous fluids, or even steroids and emergency colostomy a third of these children die. Those who survive a stormy illness will have permanent mucosal damage of both ganglionic and aganglionic bowel and an increased risk of complications such as stricture and leakage after surgery. After treatment of Hirschsprung's disease many children continue to have the problem of soiling or constipation, but this eventually clears up and over $90 \%$ of these children have normal bowel habits.

Miss Caroline M Doig is senior lecturer in paediatric surgery, Booth Hall Children's Hospital, University of Manchester.

The ABC of Colorectal Diseases has been edited by Mr D Jones, lecturer and honorary senior registrar, and Professor M H Irving, department of general surgery, Hope Hospital, Salford.

\title{
Osteogenesis imperfecta: fractures of the femur when testing for congenital dislocation of the hip
}

\author{
Colin R Paterson, Robin J Beal, John A Dent
}

Osteogenesis imperfecta must be excluded

whenever possible before routine clinical testing

for congenital dislocation of the hip
Department of Biochemical Medicine, Ninewells Hospital and Medical School, Dundee DD1 9SY Colin R Paterson, senior lecturer

\section{Department of}

Orthopaedic and Trauma

Surgery, Royal Infirmary, Dundee DD1 9ND

Robin J Beal, medical student John A Dent, senior lecturer

Correspondence to: Dr Paterson.
One important need in the management of osteogenesis imperfecta is the prevention of avoidable fractures. Each fracture leads to a period of immobilisation, and the consequent osteopenia may make further fractures more likely. This paper reports on a group of children whose initial fractures seem to have occurred as a result of clinical testing for congenital dislocation of the hip and seeks to identify ways in which these early fractures could be prevented.

\section{Case histories}

The patients were identified through the Brittle Bone Society, which maintains a register of patients in the United Kingdom and Ireland with known osteogenesis imperfecta. ${ }^{1}$ Patients were classified according to the Sillence scheme. ${ }^{2}$

Case 1-A girl was well until the age of 1 week, when her hips were examined by a paediatrician. She cried immediately but her mother was reassured. Subsequently, however, she continued to cry. excessively when nappies were changed and she was noticed not to be moving one leg. Her mother consulted her general practitioner on three occasions, and on each of these further examinations of the hips were carried out. Eventually, at the age of 4 weeks, the left leg was noted to be swollen and after referral to the casualty department bilateral femoral fractures were identified together with tibial fractures. The parents were at first questioned closely about the possibility of non-accidental injury, but eventually it was accepted that the sclerae were abnormally blue. There was no family history of osteogenesis imperfecta, but the subsequent history was typical of type IB disease.

Case 2-A boy was born by caesarean section and shortly afterwards his hips were examined without ill effects. At 6 weeks of age he was re-examined by the health visitor at the practice surgery. Immediately he cried inconsolably and the leg swelled. After rapid referral to hospital an oblique fracture of the upper third of the femur was identified. Initially nonaccidental injury was considered because of the normal sclerae and lack of a family history. Further radiology suggested osteopenia. The subsequent history was typical of osteogenesis imperfecta type IVA; with impaired growth and 14 femoral fractures over nine years.

Case 3-A previously well girl had her hips examined by a paediatrician at the age of 9 weeks. She cried immediately and the doctor commented that he felt a "grinding" in the thighs. Radiography showed bilateral fresh femoral fractures (figure). Subsequent study of the family history showed that the child's mother and grandmother were both mildly affected with osteogenesis imperfecta type IVB.

Case 4-A girl's hips were examined soon after birth without difficulty. However, at the age of 6 weeks she was taken to another hospital for a routine check, when 


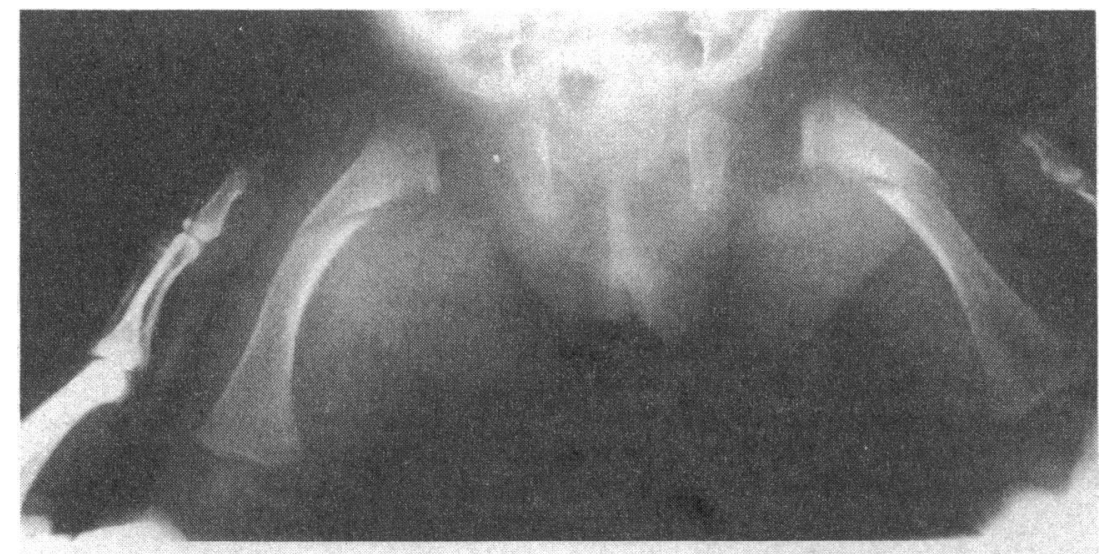

Case 3. Bilateral femoral fractures found after examination for congenital dislocation of hip

blue sclerae were noted. A second examination was carried out at that stage, after which she screamed and continued to scream for several hours. Eventually radiography disclosed a fractured left femur and an old fracture of the clavicle. Subsequently she had persisting blue-grey sclerae and an "inverted triangle" head typical of osteogenesis imperfecta type IA. She suffered only two further fractures, at ages 3 and 8 , but deformity from the previously fractured femur persisted.

Case 5-A boy was born by caesarean section because of the history of osteogenesis imperfecta type IVA in his mother and grandfather. After advice from the Brittle Bone Society the parents specifically asked both verbally and in writing that he should not be examined in the usual way for congenital dislocation of the hips. However, at the age of 2 days he was examined by a paediatric registrar. There was no immediate reaction but from that time he cried whenever he was moved. As this problem persisted he was referred back to hospital one week later. Radiographs showed a left femoral fracture together with healed fractures of both humeri and of two ribs.

Case 6-At the age of 1 day a girl's hips were examined and immediately she started screaming. She screamed for 12 hours and subsequently every time her nappy was changed over the next four or five days. At the age of 8 days she was found to have a fractured humerus. Radiography showed a fracture of the left femur with early healing. There was no family history of osteogenesis imperfecta but the subsequent history was typical of type IB disease.

Case 7-A girl whose mother, grandfather, and two aunts all had osteogenesis imperfecta type IA was radiographed for congenital hip dislocation in the von Rosen position ${ }^{3}$ at the age of 5 weeks after "clicking" had been heard in her hips. The radiographer carrying out this investigation heard audible cracks and the baby screamed immediately. Radiographs taken after this manipulation showed bilateral oblique fractures of the upper femoral shafts. She had, and continued to have, clearly abnormal sclerae. She continued to sustain fractures but grew well.

Case 8-A girl with no family history of osteogenesis imperfecta was well for the first 24 hours. Before discharge her hips were examined by a junior member of the paediatric staff and she immediately started screaming. The parents were told that she had a dislocated hip and that they should take her home, put her in double nappies, and return to the outpatient clinic two weeks later. At home the parents found that she did not move the right leg and that nappies could not be changed without her screaming. Within two days it was noted that the leg had become swollen and discoloured. Radiography then showed an oblique fracture of the upper femur. Subsequent fractures of the other femur and of the right humerus occurred over the next two months, when it was noted that the sclerae were blue and the anterior fontanelle very large.

\section{Discussion}

It is widely accepted that systematic screening of all infants for congenital dislocation of the hip prevents many cases of children presenting late with irreversible complications of the disorder. ${ }^{4}$ The standard clinical tests were introduced by Ortolani ${ }^{5}$ and Barlow. ${ }^{6}$ With both it is said that between $1 \%$ and $2 \%$ of infants show clinically detectable instability, but in many of these stabilisation occurs without treatment. It is now suggested that only between one and three infants per 1000 have residually unstable hips.

Our experience of these cases of osteogenesis imperfecta indicates that the standard clinical procedures may cause fractures and that these fractures may have long term consequences. In two of the patients (cases 2 and 6 ) there was no family history and no obvious clinical signs to suggest osteogenesis imperfecta. The paediatricians concerned could not have anticipated the difficulties that arose. In three other cases there was a family history that was ignored; in one of these the parents specifically asked that, because of the family history, the standard tests should not be done. In the remaining three patients with no family history the finding of distinctly blue or grey sclerae could have led to an early suspicion of osteogenesis imperfecta before the clinical test was carried out.

The danger of fractures when the hips of children with osteogenesis imperfecta are examined with the standard clinical tests has not previously been emphasised. There is one case report of a fractured femur at the age of 10 weeks in a preterm child with brittle bones due to copper deficiency.

How common is this problem with osteogenesis imperfecta? The number of children born each year with the disease is not known with confidence. Various estimates of its incidence have been made but all are limited by incomplete ascertainment. ${ }^{8}$ Probably the number is between 50 and 100 per million live births. For the United Kingdom this would represent between 30 and 60 new cases per year. Of these, our data imply that in about $20 \%$ the diagnosis would be obvious at birth with deformity or fracture (largely the type III disease); in a further $70 \%$ there would be blue sclerae or a family history, or both; and in some $10 \%$ there would be neither. Most infants at risk should therefore be identifiable before examination.

In seven of our cases the child was clearly distressed and screamed immediately the test was carried out. In the remaining case the signs of distress were obvious within 12 hours. In several cases the diagnosis of a fractured femur was not made as early as it should have been. In some of these cases the parents were reassured with remarks such as, "all babies cry after this test." We think that a child who is distressed after an examination of the hips should have early radiography. It is important to be aware of the possibility of fracture. Equally we think that finding a fracture after an examination of the hips should lead to the suspicion of osteogenesis imperfecta or some other brittle bone disease.

Could there be an association between osteogenesis imperfecta and congenital dislocation of the hips? Though dislocations generally are more common in osteogenesis imperfecta than in the general population, there are insufficient data either in our experience or in published reports to know whether there is an association between the two conditions. However, two cases in siblings have been reported. ${ }^{\text {T }}$ There is no reason to suppose that congenital hip dislocation is any less common in osteogenesis imperfecta than in the 
general population. It is reasonable, therefore, that these children should be tested in some way, but clearly the standard clinical tests are contraindicated. We suggest that all infants with a family history of osteogenesis imperfecta or with suggestive clinical signs, such as unusually blue sclerae or large fontanelles, should be tested by other methods. The recent improvements in ultrasound screening mean that this is probably the method of choice at present. ${ }^{10}$

We are grateful for the support of the Rehabilitation and Medical Research Trust and of the White Top Foundation. We thank members of the Brittle Bone Society for their help and Dr Susan McAllion for her part in classifying the patients.
1 Paterson CR, McAllion SJ. Osteogenesis imperfecta in the differential diagnosis of child abuse. BMF 1989;299:1451-4.

Sillence DO. Osteogenesis imperfecta: an expanding panorama of variants. Clin Orthop 1989;159:11-25.

von Rosen S. Diagnosis and treatment of congenital dislocation of the hip joint in the new-born. F Bone ft Surg 1962;44B:284-91.

Moss GD, Cartlidge PHT, Speidal BD, Chambers TL Routine examination in the neonatal period. BMF 1991;302:878-9.

in the $M$. Un serno poco Ortolani M. Un segno poco noto e sua importanza per la diagnosi precoce
prelussazione congenita dell'anca. Pediatria (Napoli) 1937;45:129-36.

prelussazione congenita dell' anca. Pediatria (Napoli) 1937;45:129-36.
Barlow TG. Early diagnosis and treatment of congenital dislocation of the hip. Barlow TG. Early diagnosis and treat
f Bone ft Surg 1962;44B:292-301.

Blumenthal I, Lealman GT, Franklyn PP. Fracture of the femur, fish odou and copper deficiency in a preterm infant. Arch Dis Child 1980;134:1155-6. Smith R, Francis MJO, Houghton GR. The britle bone syndrome: osteogenesi imperfecta. London: Butterworths, 1983.

9 du Toit SN, Weiss C. Congenital dislocation of the hip associated with osteogenesis imperfecta in male siblings. Bull Hosp ft Dis 1969;30:164-70.

10 Novick GS. Sonography in pediatric hip disorders. Radiol Clin North Am 1988;26:29-53.

(Accepted 29 April 1992)

\title{
Current Issues in Cancer
}

\section{Quality of life: philosophical question or clinical reality?}

\author{
Maurice L Slevin
}

This is the thirteenth in a series of articles examining recent developments in cancer
Imperial Cancer Research Fund, Department of Medical Oncology, St Bartholomew's Hospital, London EC1A 7BE

Maurice L Slevin, consultant physician

Series edited by: Dr G M Mead.

BMF 1992;305:466-9
Quality of life is the new catch phrase in cancer medicine. Like happiness it is one of those terms that we all understand but for which adequate definitions do not exist. The focus on quality of life for cancer patients has come with the realisation that while dramatic improvements have taken place in the treatment of the less common cancers, and while adjuvant chemotherapy has improved the cure rate for several common cancers when diagnosed and treated in the early stages, mest advanced common cancers remain incurable. In these circumstances treatment is given with the intention of providing maximum prolongation of good quality life. This, however, is not always a simple task as the treatment required for the cancer is often associated with side effects which can impair quality of life.

Cancer and its treatment often create havoc and chaos in the lives of patients and their families while other diseases such as ischaemic heart disease, which are a greater cause of death in the population, do not invoke the same degree of panic. Patients with metastatic cancer often have only minor symptoms and have to cope with the realisation that something has been growing inside them without their knowledge and while they were feeling well. This lack of symptoms may paradoxically make the illness seem more frightening and less controllable. In addition, the cause of the illness cannot usually be explained, the description of the disease is likely to be complex and confusing, and it is apparent that even the experts do not have all the answers. Furthermore, people are often told "there is nothing we can do for you," and this error is often compounded by an inaccurate prediction such as "you have six months or a year to live." It is therefore not surprising that patients with cancer of ten feel more miserable and despondent than patients with other potentially fatal illnesses and that quality of life is a much bigger issue in cancer than it is in other equally life threatening diseases.

It is now generally agreed that quality of life should be measured as an integral component of most cancer trials, particularly where treatments are given with palliative intent. ${ }^{1}$ However, this is easier said than done. Time is short in busy cancer clinics, and with increasing emphasis on trials including large numbers of patients carried out mainly in district general hospitals the logistics are formidable.

In practice quality of life data can usually be collected in only a subset of patients within a trial and particularly in those centres where specialist nurses are available. Provided randomisation takes place within each centre this should not introduce significant bias.

\section{Which instruments should we use?}

A vast array of quality of life questionnaires is currently on offer. Maguire and Selby carried out an excellent review of available measures on behalf of the Medical Research Council Cancer Therapy Committee. $^{2}$ They assessed several instruments looking at clinical utility, ease of administration, and scoring and also reliability and validity. They concluded that the best current method for assessing the key dimensions of quality of life was the Rotterdam symptom checklist. ${ }^{3}$ The hospital anxiety depression scale $^{4}$ was also particularly useful for assessing anxiety and depression.

The Rotterdam symptom checklist comprises 30 items, each rated on a 4 point scale measuring physical and psychological dimensions of quality of life. It is easy to score and can be divided into different subsets. There are two main subscales, one for physical complaints and one for psychological issues. It has been suggested that this scale does not adequately cover sexual or social dimensions of quality of life and additional physical items have been proposed for use with specific groups of cancer patients.

The hospital anxiety and depression scale was specifically designed to measure anxiety and depression in patients who were physically ill and therefore excluded somatic symptoms of depression, such as weight loss and constipation, which might also be related to the illness. It is a self rating scale and has two subscales for anxiety and depression. Its validity has been confirmed in several studies. It has the advantage of being short and easy to understand and having a simple scoring system. A score of 7 or less implies normality, 8 or 10 is borderline, and 11 or more suggests significant anxiety or depression.

These two scales thus represent the most practical and reliable measures for assessing the quality of life at this time. The European Organisation for Research and Treatment in Cancer questionnaire, ${ }^{5}$ which is currently in the final stages of development and validation, offers the possibility of a scale which is easy to score and is designed to measure key dimensions 